\title{
Likelihood Ratio Test and Non-parametric Test for Load Sharing
}

\author{
Santosh Shashikant Sutar \\ Yashwantrao Chavan School of Rural Development, Shivaji University
}

\begin{abstract}
In present article, we propose a likelihood ratio test and a non-parametric test for testing the load sharing effect observed in the two component parallel load sharing system. We have modeled the load sharing phenomenon observed in such system by the exponentiated conditional distribution function based load sharing model proposed by Sutar and Naik-Nimbalkar (2016). We have taken component baseline distribution as Weibull distribution and linear failure rate distribution. The simulation study to see the performance of proposed test procedures is reported. We analyze two data sets for illustrative purpose.
\end{abstract}

Keywords: conditional distribution, likelihood ratio test, load sharing, non-parametric test, order statistics, two-component parallel system.

\section{Introduction}

The systems in which the failure of a component increases or decreases the load on other surviving components, thereby decreasing or increasing their chances of survival are known as load sharing systems. Such systems are also referred to as dynamic reliability systems and have several practical applications. Examples of load sharing systems include fibrous composite materials, power plants, automobiles, two jet engines of an airplane among others (Liu (1998)). These load sharing models have many applications in various fields engineering (Singh and Gupta (2012)).

The earliest work on load sharing was carried by Daniels (1945). Then, many authors contribute to the load-share models, for instance, Birnbaum and Saunders (1958), Coleman (1958), Rosen (1964), Gross, Clark, and Liu (1971), Phoenix (1978), Singpurwalla (1995), Hollander and Peña (1995), Kamps (1995), Cramer and Kamps (1996), Cramer and Kamps (2001), Cramer and Kamps (2003), Lynch (1999), Drummond, Vázquez, Sánchez-Colón, Martinez-Gómez, and Hudson (2000), Durham and Lynch (2000), Kim and Kvam (2004), Kvam and Pena (2005), McCool (2006), Peña (2006), Amari, Misra, and Pham (2006), Suprasad, Krishna, and Hoang (2008), Amari and Bergman (2008), Deshpande, Dewan, and Naik-Nimbalkar (2010), Dewan and Naik-Nimbalkar (2010), Jain and Gupta (2012), Singh and Gupta (2012), Mohammad, Kalam, and Amari (2013), Gurov and Utkin (2014), Yang, Zeng, and Guo (2015), Sutar and Naik-Nimbalkar (2014), Sutar and Naik-Nimbalkar (2016), Krivtsov, Amari, and Gurevich (2018), Kim (2018) and Sutar and Naik-Nimbalkar (2019) 
some of the references cited there in.

Sutar and Naik-Nimbalkar (2016) have considered a $k$-out-of- $m$ system and proposed load sharing model through exponentiated conditional distributions of the order statistics. It leads to a new family of multivariate distributions for the ordered r.v.'s, which is different from the one introduced by Kamps (1995) and Cramer and Kamps (1996), Cramer and Kamps (2001) for sequential order statistics. In the present article, we consider a model for $k$-out-of- $m$ load sharing systems proposed by Sutar and Naik-Nimbalkar (2016). We consider a two component parallel load sharing system and propose the two tests namely the likelihood ratio test (LRT) and the non-parametric test for testing the load sharing effect observed in such system. The performance of the proposed test procedures is also evaluated with extensive simulation study. The rest of this article is organized as follows.

In Section 2, we restate the load sharing model for a two component parallel system, in a general setup and also when the initial lifetimes of the components having Weibull and linear failure rate distributions. For the general setup, we propose a LRT and non-parametric tests to test the hypothesis that, the component failures occurs independently against the alternative that they show the load sharing phenomenon in Section 3. In Section 4, simulation study showing the performance of the test procedures is reported. We report the illustrations in Section 5 and conclusions are given in the last section.

\section{The model and the associated parameter estimation procedure}

\subsection{General setup}

We consider a two component parallel system with $U_{1}, U_{2}$ as the component lifetimes, being independent and identically distributed (i.i.d.) with common probability density function (p.d.f.) $f(\cdot ; \underline{\lambda})$ where $\underline{\lambda}$ may be scalar or vector valued parameter. Let $F(\cdot ; \underline{\lambda})$ and $\bar{F}(\cdot ; \underline{\lambda})$ denote corresponding cumulative distribution function (c.d.f.) and survival function (s.f.). Let $X=\min \left(U_{1}, U_{2}\right)$ denote the first failure time and $Y=\max \left(U_{1}, U_{2}\right)$ denote the second failure time. The density of $X$ is

$$
k_{X}(x, \underline{\lambda})=2 f(x ; \underline{\lambda}) \bar{F}(x ; \underline{\lambda}), \quad x>0 .
$$

According to load sharing model proposed by Sutar and Naik-Nimbalkar (2016), the conditional density of $Y$ given $X=x$ is

$$
k_{Y \mid X=x}(y ; \beta, \underline{\lambda})=\beta\left[1-\frac{\bar{F}(y ; \underline{\lambda})}{\bar{F}(x ; \underline{\lambda})}\right]^{\beta-1} \frac{f(y ; \underline{\lambda})}{\bar{F}(x ; \underline{\lambda})}, 0<x<y<\infty, \beta>0 .
$$

and the joint density of $(X, Y)$ is

$$
k(x, y ; \beta, \underline{\lambda})=2 \beta\left[1-\frac{\bar{F}(y ; \underline{\lambda})}{\bar{F}(x ; \underline{\lambda})}\right]^{\beta-1} f(x ; \underline{\lambda}) f(y ; \underline{\lambda}), 0<x<y<\infty, \beta>0 .
$$

We note that, $\beta=1$ implies independent setup whereas $\beta \neq 1$ implies dependent or load sharing setup. Let $\left\{\left(x_{i}, y_{i}\right), i=1,2, \cdots, n\right\}$ be a random sample of size $n$ from the joint density given in (2). We use the two step procedure proposed by Sutar and Naik-Nimbalkar (2016) for estimating the unknown parameters. This procedure is described as follow.

Step (i): Estimate $\underline{\lambda}$ based on observations on $X$, the first failure time.

That is we can estimate $\underline{\lambda}$ by maximizing the following $\log$-likelihood, $\log L_{X}(\underline{\lambda})$, based on $X$,

$$
\log L_{X}(\underline{\lambda})=n \log 2+\sum_{i=1}^{n} \log f\left(x_{i} ; \underline{\lambda}\right)+\sum_{i=1}^{n} \log \left(\bar{F}\left(x_{i} ; \underline{\lambda}\right)\right) .
$$


Step (ii): Estimate $\beta$ by using conditional distribution of $Y$ given $X=x$ and by plugging in the estimates of $\underline{\lambda}$ as $\underline{\hat{\lambda}}$ (say), obtained in step (i).

Thus, the estimate $\hat{\beta}$ of $\beta$ is given by

$$
\hat{\beta}=\left[-\frac{1}{n} \sum_{i=1}^{n} \log \left(1-\frac{\bar{F}\left(y_{i} ; \underline{\hat{\lambda}}\right)}{\bar{F}\left(x_{i} ; \underline{\hat{\lambda}}\right)}\right)\right]^{-1} .
$$

In the next subsection, we discuss the same setup when baseline distributions are Weibull and LFR.

\subsection{Weibull distribution}

We consider a two component parallel load sharing system, with the component lifetimes being i.i.d. Weibull r.v.'s with shape parameter $\lambda$ and scale parameter $\theta$, with p.d.f.

$$
f\left(u_{i}\right)=\theta \lambda u_{i}^{\lambda-1} e^{-\theta u_{i}^{\lambda}}, \quad u_{i}>0, \lambda>0, \theta>0, i=1,2 .
$$

Then, the first failure time, $\mathrm{X}$ is a Weibull random variable with shape parameter $\lambda$ and scale parameter $2 \theta$. The conditional density of $\mathrm{Y}$ given $X=x$ as

$$
k_{Y \mid X=x}(y)=\beta \lambda \theta y^{\lambda-1}\left\{1-e^{-\theta\left(y^{\lambda}-x^{\lambda}\right)}\right\}^{\beta-1} e^{-\theta\left(y^{\lambda}-x^{\lambda}\right)}, 0<x<y<\infty, \lambda>0, \theta>0, \beta>0 .
$$

The joint density of $(\mathrm{X}, \mathrm{Y})$ can be written as

$$
k(x, y)=2 \beta \lambda^{2} \theta^{2}(x y)^{\lambda-1}\left\{1-e^{-\theta\left(y^{\lambda}-x^{\lambda}\right)}\right\}^{\beta-1} e^{-\theta\left(y^{\lambda}-3 x^{\lambda}\right)}, 0<x<y<\infty, \lambda>0, \theta>0, \beta>0 .
$$

\section{Estimation procedure}

We adopt two step estimation procedure proposed by Sutar and Naik-Nimbalkar (2016) and the two step estimation procedure for estimating $(\lambda, \theta, \beta)$ is as follow.

\section{Step (i): Estimation of $\lambda$ and $\theta$}

The log-likelihood based on $\mathrm{X}$ as

$$
\log L_{X}=n \log 2+n \log \theta+n \log \lambda+(\lambda-1) \sum_{i=1}^{n} \log x_{i}-2 \theta \sum_{i=1}^{n} x_{i}^{\lambda} .
$$

The maximum likelihood estimates $(\hat{\lambda}, \hat{\theta})$ of $(\lambda, \theta)$ by solving the following log-likelihood equations by iterative procedures such as Newton-Raphson method,

$$
\begin{gathered}
\frac{\partial \log L_{X}}{\partial \theta}=\frac{n}{\theta}-2 \sum_{i=1}^{n} x_{i}^{\lambda}=0 \\
\frac{\partial \log L_{X}}{\partial \lambda}=\frac{n}{\lambda}+\sum_{i=1}^{n} \log x_{i}-2 \theta \sum_{i=1}^{n}\left(\log x_{i}\right) x_{i}^{\lambda}=0 .
\end{gathered}
$$

\section{Step (ii): Estimation of $\beta$}

Once we get estimates of $\lambda$ and $\theta$, the estimate of $\beta$ follows from the expression (4), thus

$$
\hat{\beta}=\left[-\frac{1}{n} \sum_{i=1}^{n} \log \left\{1-e^{-\hat{\theta}\left(y_{i}^{\hat{\lambda}}-x_{i}^{\hat{\lambda}}\right)}\right\}\right]^{-1} .
$$




\subsection{LFR case}

We consider a two component parallel load sharing system, with the component lifetimes being i.i.d. LFR r.v.'s with parameters $\lambda_{1}, \lambda_{2}$, with p.d.f.

$$
f\left(u_{i}\right)=\left(\lambda_{1}+2 \lambda_{2} u_{i}\right) \exp \left\{-\left(\lambda_{1} u_{i}+\lambda_{2} u_{i}^{2}\right)\right\}, \quad u_{i}>0, \lambda_{1}>0, \lambda_{2} \geq 0, i=1,2 .
$$

Then, $\mathrm{X}$ has the LFR distribution with parameters $2 \lambda_{1}=\eta_{1}$ (say) and $2 \lambda_{2}=\eta_{2}$ (say), with p.d.f.

$$
k_{X}(x)=\left\{\eta_{1}+2 \eta_{2} x\right\} e^{-\left\{\eta_{1} x+\eta_{2} x^{2}\right\}}, \quad x>0, \eta_{1}>0, \eta_{2}>0 .
$$

The conditional density of $Y$ given $X=x$ as

$$
k_{Y \mid X=x}(y)=\beta\left\{\lambda_{1}+2 \lambda_{2} y\right\}\left\{1-e^{-\left\{\lambda_{1}(y-x)+\lambda_{2}\left(y^{2}-x^{2}\right)\right\}}\right\}^{\beta-1} e^{-\left\{\lambda_{1}(y-x)+\lambda_{2}\left(y^{2}-x^{2}\right)\right\}},
$$

The joint density of $(X, Y)$ as

$$
\begin{array}{r}
k(x, y)=2 \beta\left\{\lambda_{1}+2 \lambda_{2} x\right\}\left\{\lambda_{1}+2 \lambda_{2} y\right\}\left\{1-e^{-\left\{\lambda_{1}(y-x)+\lambda_{2}\left(y^{2}-x^{2}\right)\right\}}\right\}^{\beta-1} e^{-\left\{\lambda_{1}(x+y)+\lambda_{2}\left(x^{2}+y^{2}\right)\right\}}, \\
0<x<y<\infty, \lambda_{1}, \lambda_{2}, \beta>0 .
\end{array}
$$

The two step estimation procedure

\section{Step (i): Estimation of $\lambda_{1}$ and $\lambda_{2}$}

Since, $\mathrm{X}$ has the LFR distribution with parameters $\eta_{1}=2 \lambda_{1}$ and $\eta_{2}=2 \lambda_{2}$, we employ same step of Sutar and Naik-Nimbalkar (2016) for finding the estimates of $\left(\eta_{1}, \eta_{2}\right)$. Specifically, letting $\eta_{1}^{(p)}$ and $\eta_{2}^{(p)}$ denote the estimates of $\eta_{1}$ and $\eta_{2}$, respectively, at the $p^{t h}$ stage of iteration, $\eta_{1}^{(p+1)}$ and $\eta_{2}^{(p+1)}$ are obtained as

$$
\begin{aligned}
& \eta_{1}^{(p+1)}=\left\{\frac{1}{\eta_{1}^{(p)}}+n^{-1}\left(\sum_{i=1}^{n} x_{i}-\sum_{i=1}^{n} \frac{1}{\left(\eta_{1}^{(p)}+2 \eta_{2}^{(p)} x_{i}\right)}\right)\right\}^{-1}, \\
& \eta_{2}^{(p+1)}=\left\{\frac{1}{\eta_{2}^{(p)}}+n^{-1}\left(\sum_{i=1}^{n} x_{i}^{2}-\sum_{i=1}^{n} \frac{2 x_{i}}{\left(\eta_{1}^{(p)}+2 \eta_{2}^{(p)} x_{i}\right)}\right)\right\}^{-1} .
\end{aligned}
$$

Once we get the estimates $\left(\widehat{\eta}_{1}, \widehat{\eta}_{2}\right)$ of $\left(\eta_{1}, \eta_{2}\right)$, we can obtain estimates of $\left(\lambda_{1}, \lambda_{2}\right)$ as $\left(\widehat{\lambda}_{1}=\frac{\widehat{\eta}_{1}}{2}, \widehat{\lambda}_{2}=\frac{\widehat{\eta}_{2}}{2}\right)$.

\section{Step (ii): Estimation of $\beta$}

We estimate $\beta$ from the expression (4) as

$$
\hat{\beta}=\left[-\frac{1}{n} \sum_{i=1}^{n} \log \left\{1-e^{-\left\{\hat{\lambda}_{1}\left(y_{1}-x_{i}\right)+\hat{\lambda}_{2}\left(y_{i}^{2}-x_{i}^{2}\right)\right\}}\right\}\right]^{-1} .
$$

In the next section, we propose a test procedures for testing the load sharing effect.

\section{The proposed test procedures}

We consider a two component parallel load sharing system and our interest is to test whether the lifetimes of the system components are independent against the alternative that there exits a load sharing phenomenon. We propose a LRT and non-parametric test for testing the load sharing effect. We set the null hypothesis as $H_{0}: \beta=1$, i.e. the first failure does not 
affect the lifetime of the second component and the alternative hypothesis as $H_{1}: \beta \neq 1$, i.e. the first failure affects (stochastically changes) the lifetime of the second component. The data consist of $n$ i.i.d. pairs of ordered component lifetimes and is denote by, $(\underline{\mathbf{x}}, \underline{\mathbf{y}})=$ $\left\{\left(x_{i}, y_{i}\right): x_{i} \leq y_{i} ; i=1,2, \ldots, n\right\}$. The joint density of $(X, Y)$ in terms of conditional of $Y$ given $X=x$ and marginal density of $X$ can be written as

$$
k_{(X, Y)}(x, y ; \beta, \underline{\lambda})=k_{Y \mid X=x}(y ; \beta, \underline{\lambda}) k_{X}(x ; \underline{\lambda}), x<y, \beta>0, \underline{\lambda}>\underline{0} .
$$

Then the complete likelihood, $L_{(X, Y)}$, based on data $(\underline{\mathbf{x}}, \underline{\mathbf{y}})$ is given by

$$
L_{(X, Y)}=\prod_{i=i}^{n} k_{Y_{i} \mid X_{i}=x_{i}}\left(y_{i} ; \beta, \underline{\lambda}\right) k_{X_{i}}\left(x_{i} ; \underline{\lambda}\right)=L_{Y \mid X=x}(\beta, \underline{\lambda} ; \underline{\mathbf{x}}, \underline{\mathbf{y}}) L_{X}(\underline{\lambda}, \underline{\mathbf{x}}),
$$

where $L_{Y \mid X=x}(\beta, \underline{\lambda} ; \underline{\mathbf{x}}, \underline{\mathbf{y}})=\prod_{i=i}^{n} k_{Y_{i} \mid X_{i}=x_{i}}\left(y_{i} ; \beta, \underline{\lambda}\right)$, is the likelihood based on conditional density of $Y$ given $X=\bar{x}$ and $L_{X}(\underline{\lambda}, \underline{\mathbf{x}})=\prod_{i=i}^{n} k_{X_{i}}\left(x_{i} ; \underline{\lambda}\right)$, is the likelihood based on marginal density of $X$.

In the next subsections, we propose two test procedures for testing the load sharing effect.

\subsection{Likelihood ratio test}

The LRT statistic for testing $H_{0}$ against $H_{1}$ is

$$
\mu^{*}(\underline{\mathbf{x}}, \underline{\mathbf{y}})=\frac{\sup _{\Theta_{0}}\left\{L_{Y \mid X=x}(\beta, \underline{\lambda} ; \underline{\mathbf{x}}, \underline{\mathbf{y}}) L_{X}(\underline{\lambda}, \underline{\mathbf{x}})\right\}}{\sup _{\Theta}\left\{L_{Y \mid X=x}(\beta, \underline{\lambda} ; \underline{\mathbf{x}}, \underline{\mathbf{y}}) L_{X}(\underline{\lambda}, \underline{\mathbf{x}})\right\}},
$$

where $\Theta_{0}=\{(\beta ; \underline{\lambda}) \mid \beta=1, \underline{\lambda} \geq 0\}, \Theta_{1}=\{(\beta ; \underline{\lambda}) \mid \beta \neq 1, \underline{\lambda} \geq 0\}$ and $\Theta=\left\{\Theta_{0} \cup \Theta_{1}\right\}=$ $\{(\beta ; \underline{\lambda}) \mid \beta>0, \underline{\lambda} \geq \underline{0}\}$.

We modify the usual LRT statistic by estimating $\beta$ and $\underline{\lambda}$ using the two step procedure discussed in the previous section. Let these estimators be denoted by $\hat{\beta}$ and $\underline{\hat{\lambda}}$. We also note that, $\sup _{\Theta_{0}}\left\{L_{X}(\underline{\lambda}, \underline{\mathbf{x}})\right\}=\sup _{\Theta}\left\{L_{X}(\underline{\lambda}, \underline{\mathbf{x}})\right\}$ as the marginal density of $X$ remains the same in both $H_{0}$ and $H_{1}$ setups. Thus, the test statistic can be written as

$$
\mu(\underline{\mathbf{x}}, \underline{\mathbf{y}})=\frac{\sup _{\Theta_{0}}\left\{L_{Y \mid X=x}(\beta, \underline{\lambda} ; \underline{\mathbf{x}}, \underline{\mathbf{y}})\right\}}{\sup _{\Theta}\left\{L_{Y \mid X=x}(\beta, \underline{\lambda} ; \underline{\mathbf{x}}, \underline{\mathbf{y}})\right\}}=\frac{\left.L_{Y \mid X=x}(\beta, \underline{\lambda} ; \underline{\mathbf{x}}, \underline{\mathbf{y}})\right|_{\left(\beta_{0}, \hat{\underline{\lambda}}\right)}}{\left.L_{Y \mid X=x}(\beta, \underline{\lambda} ; \underline{\mathbf{x}}, \underline{\mathbf{y}})\right|_{(\hat{\beta}, \hat{\lambda})}} .
$$

Lemma 1. $\hat{\lambda}$ is consistent estimator of $\lambda_{0}$ (true parameter). That is, as $n \rightarrow \infty$,

$$
\hat{\lambda} \stackrel{p}{\rightarrow} \lambda_{0} .
$$

Proof. The consistency of $\hat{\lambda}$ follows from the Cramer-Huzurbazar theorem (Kale (2005)). As the all regularity conditions of the Cramer-Huzurbazar theorem is satisfied by the density $k(x, \lambda)$ of, the first failure, $X$. We claim that $k(x)=2 f(x ; \lambda) \bar{F}(x ; \lambda)$ belongs to the Cramer class.

Lemma 2. $\hat{\beta}$ is consistent estimator of $\beta_{0}$ (true parameter). That is, as $n \rightarrow \infty$,

$$
\hat{\beta} \stackrel{p}{\rightarrow} \beta_{0} \text {. }
$$

Proof. By using Taylor series expansion of $\left.\frac{\partial \log L_{Y \mid X=x}(\beta, \lambda)}{\partial \beta}\right|_{(\hat{\beta}, \hat{\lambda})}$ around $\left(\beta_{0}, \hat{\lambda}\right)$, we get

$$
\left.\frac{\partial \log L_{Y \mid X=x}(\beta, \lambda)}{\partial \beta}\right|_{(\hat{\beta}, \hat{\lambda})}=\left.\frac{\partial \log L_{Y \mid X=x}(\beta, \lambda)}{\partial \beta}\right|_{\left(\beta_{0}, \hat{\lambda}\right)}+\left.\left(\hat{\beta}-\beta_{0}\right) \frac{\partial^{2} \log L_{Y \mid X=x}(\beta, \lambda)}{\partial \beta^{2}}\right|_{\left(\beta^{*}, \hat{\lambda}\right)},
$$


where, $\beta^{*}=a \beta_{0}+(1-a) \hat{\beta}, a \in(0,1)$.

$\left.\operatorname{As} \frac{\partial \log L_{Y \mid X=x}(\beta, \lambda)}{\partial \beta}\right|_{(\hat{\beta}, \hat{\lambda})}=0,\left.\quad \frac{\partial^{2} \log L_{Y \mid X=x}(\beta, \lambda)}{\partial \beta^{2}}\right|_{\left(\beta^{*}, \hat{\lambda}\right)}=-\frac{n}{\left(\beta^{*}\right)^{2}}$,

we have

$$
\left(\hat{\beta}-\beta_{0}\right)=\beta^{* 2}\left\{\left.\frac{1}{n} \frac{\partial \log L_{Y \mid X=x}(\beta, \lambda)}{\partial \beta}\right|_{\left(\beta_{0}, \hat{\lambda}\right)}\right\} .
$$

Now, we expand $\left.\frac{1}{n} \frac{\partial \log L_{Y \mid X=x}(\beta, \lambda)}{\partial \beta}\right|_{\left(\beta_{0}, \hat{\lambda}\right)}$ around $\left(\beta_{0}, \lambda_{0}\right)$ by using Taylor series expansion as

$$
\begin{array}{r}
\left.\frac{1}{n} \frac{\partial \log L_{Y \mid X=x}(\beta, \lambda)}{\partial \beta}\right|_{\left(\beta_{0}, \hat{\lambda}\right)}=\left.\frac{1}{n} \frac{\partial \log L_{Y \mid X=x}(\beta, \lambda)}{\partial \beta}\right|_{\left(\beta_{0}, \lambda_{0}\right)} \\
+\left.\frac{\left(\hat{\lambda}-\lambda_{0}\right)}{n} \frac{\partial^{2} \log L_{Y \mid X=x}(\beta, \lambda)}{\partial \beta \partial \lambda}\right|_{\left(\beta_{0}, \lambda^{*}\right)},
\end{array}
$$

where $\lambda^{*}=b \lambda_{0}+(1-b) \hat{\lambda}, b \in(0,1)$.

Consider first term,

$$
\left.\frac{1}{n} \frac{\partial \log L_{Y \mid X=x}(\beta, \lambda)}{\partial \beta}\right|_{\left(\beta_{0}, \lambda_{0}\right)}=\left.\frac{1}{n} \sum_{i=1}^{n} \frac{\partial \log k_{Y_{i} \mid X_{i}=x_{i}}\left(y_{i} ; \beta, \lambda\right)}{\partial \beta}\right|_{\left(\beta_{0}, \lambda_{0}\right)},
$$

where $k_{Y_{i} \mid X_{i}=x_{i}}\left(y_{i} ; \beta, \lambda\right) ; i=1,2, \cdots, n$ is the conditional density of $Y_{i}$ given $X_{i}=x_{i}$ and is defined as in (1). Let $U_{i}=\left.\frac{\partial \log h_{Y_{i} \mid X_{i}=x_{i}}\left(y_{i} ; \beta, \lambda\right)}{\partial \beta}\right|_{\left(\beta_{0}, \lambda_{0}\right)}$. But as $\left(X_{i}, Y_{i}\right)$ are i.i.d., we can claim that $U_{i}$ 's are also i.i.d. with $E\left(U_{1}\right)=0$. Thus, by weak law of large numbers,

$$
\left.\frac{1}{n} \frac{\partial \log L_{Y \mid X=x}(\beta, \lambda)}{\partial \beta}\right|_{\left(\beta_{0}, \lambda_{0}\right)} \stackrel{p}{\rightarrow} 0 .
$$

Consider second term,

$$
\left.\frac{(\hat{\lambda}-\lambda)}{n} \frac{\partial^{2} \log L_{Y \mid X=x}(\beta, \lambda)}{\partial \beta \partial \lambda}\right|_{\left(\beta_{0}, \lambda^{*}\right)} .
$$

As $\hat{\lambda}$ is consistent for $\lambda$ and $\lambda^{*}=b \lambda_{0}+(1-b) \hat{\lambda}, b \in(0,1)$, we have $\lambda^{*} \stackrel{p}{\rightarrow} \lambda_{0}$. Also we know that if $m(\cdot)$ is continuous function then, by invariance property of consistent estimator, we have $m\left(\lambda^{*}\right) \stackrel{p}{\rightarrow} m\left(\lambda_{0}\right)$. Therefore, we can claim that, $\left.\frac{1}{n} \frac{\partial^{2} \log L_{Y \mid X=x}(\beta, \lambda)}{\partial \beta \partial \lambda}\right|_{\left(\beta_{0}, \lambda^{*}\right)}$ is bounded in probability. Thus, by using above arguments and from Lehmma 1 , we have $\left(\hat{\beta}-\beta_{0}\right) \stackrel{p}{\rightarrow} 0$.

Lemma 3. As $n \rightarrow \infty$,

$$
-\left.\frac{1}{n} \frac{\partial^{2} \log L_{Y \mid X=x}(\beta, \lambda)}{\partial \beta^{2}}\right|_{(\hat{\beta}, \hat{\lambda})} \stackrel{p}{\rightarrow} I_{Y \mid x}\left(\beta_{0}, \lambda_{0}\right),
$$

where $I_{Y \mid x}\left(\beta_{0}, \lambda_{0}\right)=E\left[-\left.\frac{\partial^{2} \log h_{Y \mid X}(Y ; \beta, \lambda)}{\partial \beta^{2}}\right|_{\left(\beta_{0}, \lambda_{0}\right)}\right],\left.\frac{\partial^{2} \log L_{Y \mid X}(Y ; \beta, \lambda)}{\partial \beta^{2}}\right|_{\left(\hat{\beta}, \lambda_{0}\right)}=\frac{-n}{\hat{\beta}^{2}}$.

Proof. As $\hat{\beta} \stackrel{p}{\rightarrow} \beta_{0}$ and $\beta_{0}>0$, we have the result

$$
-\left.\frac{1}{n} \frac{\partial^{2} \log L_{Y \mid X=x}(\beta, \lambda)}{\partial \beta^{2}}\right|_{\left(\beta_{0}, \hat{\lambda}\right)} \stackrel{p}{\rightarrow} I_{Y \mid X=x}\left(\beta_{0}, \lambda_{0}\right)=\frac{1}{\beta_{0}^{2}}(<\infty) .
$$


Lemma 4. Asymptotic normality of $\hat{\beta}$. That is

$$
\sqrt{n}\left(\hat{\beta}-\beta_{0}\right) \stackrel{D}{\rightarrow} N\left(0, \beta_{0}^{2}\right) .
$$

Proof. By using Taylor series expansion, we expand $\left.\frac{\partial \log L_{Y \mid X=x}(\beta, \lambda)}{\partial \beta}\right|_{(\hat{\beta}, \hat{\lambda})}$ around $\left(\beta_{0}, \lambda_{0}\right)$ as

$$
\begin{gathered}
\left.\frac{\partial \log L_{Y \mid X=x}(\beta, \lambda)}{\partial \beta}\right|_{(\hat{\beta}, \hat{\lambda})}=\left.\frac{\partial \log L_{Y \mid X=x}(\beta, \lambda)}{\partial \beta}\right|_{\left(\beta_{0}, \lambda_{0}\right)}+\left.\left(\hat{\beta}-\beta_{0}\right) \frac{\partial^{2} \log L_{Y \mid X=x}(\beta, \lambda)}{\partial \beta^{2}}\right|_{\left(\beta_{0}, \lambda_{0}\right)} \\
\left.\quad\left(\hat{\lambda}-\lambda_{0}\right) \frac{\partial^{2} \log L_{Y \mid X=x}(\beta, \lambda)}{\partial \beta \partial \lambda}\right|_{\left(\beta_{0}, \lambda_{0}\right)}+\left.\frac{\left(\hat{\beta}-\beta_{0}\right)^{2}}{2 !} \frac{\partial^{3} \log L_{Y \mid X=x}(\beta, \lambda)}{\partial \beta^{3}}\right|_{\left(\beta^{*}, \lambda^{*}\right)} \\
+\left.\frac{\left(\hat{\lambda}-\lambda_{0}\right)\left(\hat{\beta}-\beta_{0}\right)}{2 !} \frac{\partial^{3} \log L_{Y \mid X=x}(\beta, \lambda)}{\partial \beta^{2} \partial \lambda}\right|_{\left(\beta^{*}, \lambda^{*}\right)}+\left.\frac{\left(\hat{\lambda}-\lambda_{0}\right)^{2}}{2 !} \frac{\partial^{3} \log L_{Y \mid X=x}(\beta, \lambda)}{\partial \beta^{3}}\right|_{\left(\beta^{*}, \lambda^{*}\right)},
\end{gathered}
$$

where, $\beta^{*}=a \beta_{0}+(1-a) \hat{\beta}, a \in(0,1)$ and $\lambda^{*}=b \lambda_{0}+(1-b) \hat{\lambda}, b \in(0,1)$.

By using the following results

$$
\begin{aligned}
& \left.\frac{\partial \log L_{Y \mid X=x}(\beta, \lambda)}{\partial \beta}\right|_{(\hat{\beta}, \hat{\lambda})}=0,-\left.\frac{1}{n} \frac{\partial^{2} \log L_{Y \mid X=x}(\beta, \lambda)}{\partial \beta^{2}}\right|_{\left(\beta_{0}, \lambda_{0}\right)}=\frac{1}{\beta_{0}^{2}}(<\infty), \\
& -\left.\frac{1}{n} \frac{\partial^{3} \log L_{Y \mid X=x}(\beta, \lambda)}{\partial \beta^{3}}\right|_{\left(\beta^{*}, \lambda^{*}\right)}=\frac{2}{\beta^{* 3}}(<\infty),\left.\frac{1}{n} \frac{\partial^{3} \log L_{Y \mid X=x}(\beta, \lambda)}{\partial \beta^{2} \partial \lambda}\right|_{\left(\beta_{0}, \lambda_{0}\right)}=0, \\
& \left.\frac{\partial^{3} \log L_{Y \mid X=x}(\beta, \lambda)}{\partial \lambda^{2} \partial \beta}\right|_{\left(\beta_{0}, \lambda_{0}\right)}=\left.\frac{\partial^{3} \log L_{Y \mid X=x}(\beta, \lambda)}{\partial \lambda \partial \beta^{2}}\right|_{\left(\beta_{0}, \lambda_{0}\right)}=0, \hat{\beta} \stackrel{p}{\rightarrow} \beta_{0}, \hat{\lambda} \stackrel{p}{\rightarrow} \lambda_{0},
\end{aligned}
$$

the expression (17) becomes

$$
\sqrt{n}\left(\hat{\beta}-\beta_{0}\right) \stackrel{d}{=} \beta_{0}^{2}\left(\left.\frac{1}{\sqrt{n}} \frac{\partial \log L_{Y \mid X=x}(\beta, \lambda)}{\partial \beta}\right|_{\left(\beta_{0}, \lambda_{0}\right)}\right) .
$$

Define, $U_{i}=\left.\frac{\partial \log k_{Y_{i} \mid X_{i}=x_{i}}\left(y_{i} ; \beta, \lambda\right)}{\partial \beta}\right|_{\left(\beta_{0}, \lambda_{0}\right)}$. As $\left(X_{i}, Y_{i}\right)$ are i.i.d. pairs, we claim that the $U_{i}$ 's are also i.i.d. with

$$
E\left(U_{1}\right)=0, V\left(U_{1}\right)=E\left[-\left.\frac{\partial^{2} \log k_{Y \mid X}(y, \beta, \lambda)}{\partial \beta^{2}}\right|_{\left(\beta_{0}, \lambda_{0}\right)}\right]=I_{Y \mid X=x}\left(\beta_{0}, \lambda_{0}\right)=\frac{1}{\beta_{0}^{2}}(<\infty) .
$$

By the central limit theorem (CLT), we have

$$
\frac{\sum_{i=1}^{n} U_{i}-n E\left(U_{1}\right)}{\sqrt{n V\left(U_{1}\right)}} \stackrel{L}{\rightarrow} Z \sim N(0,1) .
$$

That is

$$
\frac{1}{\sqrt{n}} \sum_{i=1}^{n} U_{i} \stackrel{L}{\rightarrow} Z \sim N\left(0, \frac{1}{\beta_{0}^{2}}\right)
$$

By Slutsky's theorem on convergence of random variables, we have the following result

$$
\sqrt{n}\left(\hat{\beta}-\beta_{0}\right) \stackrel{L}{\rightarrow} Z \sim N\left(0, \beta_{0}^{2}\right) .
$$

Theorem 1. Under the Cramer regularity conditions (Cramer (1946)), the asymptotic null distribution of the test statistic, $-2 \log \mu(\underline{\mathbf{X}}, \underline{\mathbf{Y}})$ is $\chi^{2}$ distribution with 1 degree of freedom. 
Proof. The joint density of $(X, Y)$ can be written as

$$
k(x, y)=k(y \mid x ; \beta, \underline{\lambda}) k(x ; \underline{\lambda}), x<y, \beta>0 .
$$

We assume that the joint density $k(x, y)$ of $(X, Y)$ satisfies the usual regularity conditions. For shake of simplicity, we take $\underline{\lambda}=\lambda$ i.e. scalar parameter and note that the same steps can be extended to $\underline{\lambda}$. The conditional density of $\mathrm{Y}$ given $X=x$ is

$$
k_{Y \mid X=x}(y)=\beta\left[1-\frac{\bar{F}(y ; \lambda)}{\bar{F}(x ; \lambda)}\right]^{\beta-1} \frac{f(y ; \lambda)}{\bar{F}(x ; \lambda)}, \quad x<y, \beta>0 .
$$

Thus,

$$
\frac{\partial \log k_{Y \mid X=x}(y)}{\partial \beta}=\frac{1}{\beta}+\log \left[1-\frac{\bar{F}(y ; \lambda)}{\bar{F}(x ; \lambda)}\right], \frac{\partial^{2} \log k_{Y \mid X=x}(y)}{\partial \beta^{2}}=\frac{-1}{\beta^{2}} .
$$

We estimate $\lambda$ from the marginal distribution of $\mathrm{X}$ and $\beta$ from conditional distribution of $Y$ given $X=x$ by plugging the estimate $\hat{\lambda}$ of $\lambda$.

From (11), we can write,

$$
-2 \log \mu(\underline{\mathbf{x}}, \underline{\mathbf{y}})=\left.2 \log L_{Y \mid X=x}(\beta, \lambda)\right|_{(\hat{\beta}, \hat{\lambda})}-\left.2 \log L_{Y \mid X=x}(\beta, \lambda)\right|_{\left(\beta_{0}, \hat{\lambda}\right)} .
$$

Through out the proof $\beta_{0}=1$, which is the value of $\beta$ under $H_{0}$. Let $\lambda=\lambda_{0}$ denote the true parameter. Now, we expand $\left.2 \log L_{Y \mid X=x}(\beta, \lambda)\right|_{\left(\beta_{0}, \hat{\lambda}\right)}$ around $(\hat{\beta}, \hat{\lambda})$ by using Taylor series as

$$
\begin{aligned}
& \left.2 \log L_{Y \mid X=x}(\beta, \lambda)\right|_{\left(\beta_{0}, \hat{\lambda}\right)}=\left.2 \log L_{Y \mid X=x}(\beta, \lambda)\right|_{(\hat{\beta}, \hat{\lambda})}+\left.2\left(\beta_{0}-\hat{\beta}\right) \frac{\partial \log L_{Y \mid X=x}(\beta, \lambda)}{\partial \beta}\right|_{(\hat{\beta}, \hat{\lambda})} \\
& \quad+\left.\frac{2\left(\beta_{0}-\hat{\beta}\right)^{2}}{2 !} \frac{\partial^{2} \log L_{Y \mid X=x}(\beta, \lambda)}{\partial \beta^{2}}\right|_{(\hat{\beta}, \hat{\lambda})}+\left.\frac{2\left(\beta_{0}-\hat{\beta}\right)^{3}}{3 !} \frac{\partial^{3} \log L_{Y \mid X=x}(\beta, \lambda)}{\partial \beta^{3}}\right|_{(\beta *, \hat{\lambda})} .
\end{aligned}
$$

By using (20), equation (19) becomes

$$
\begin{aligned}
& -2 \log \mu(\underline{\mathbf{x}}, \underline{\mathbf{y}})=-\left.2\left(\beta_{0}-\hat{\beta}\right) \frac{\partial \log L_{Y \mid X=x}(\beta, \lambda)}{\partial \beta}\right|_{(\hat{\beta}, \hat{\lambda})} \\
& +\left(\hat{\beta}-\beta_{0}\right)^{2}\left[-\left.\frac{\partial^{2} \log L_{Y \mid X=x}(\beta, \lambda)}{\partial \beta^{2}}\right|_{(\hat{\beta}, \hat{\lambda})}\right]+\left.\frac{2\left(\beta_{0}-\hat{\beta}\right)^{3}}{3 !} \frac{\partial^{3} \log L_{Y \mid X=x}(\beta, \lambda)}{\partial \beta^{3}}\right|_{(\beta *, \hat{\lambda})} .
\end{aligned}
$$

By using the following results $\left.\frac{\partial \log L_{Y \mid X=x}(\beta, \lambda)}{\partial \beta}\right|_{(\hat{\beta}, \hat{\lambda})}=0,-\left.\frac{\partial^{2} \log L_{Y \mid X=x}(\beta, \lambda)}{\partial \beta^{2}}\right|_{(\hat{\beta}, \hat{\lambda})}=\frac{n}{\hat{\beta}^{2}}$, $-\left.\frac{\partial^{3} \log L_{Y \mid X=x}(\beta, \lambda)}{\partial \beta^{3}}\right|_{(\hat{\beta}, \hat{\lambda})}=-\frac{2 n}{\hat{\beta}^{3}}$, the expression $(21)$ becomes

$$
-2 \log \mu(\underline{\mathbf{x}}, \underline{\mathbf{y}})=\frac{\left[\sqrt{n}\left(\hat{\beta}-\beta_{0}\right)\right]^{2}}{\hat{\beta}^{2}}+R_{n},
$$

where, $R_{n}=\frac{2 n\left(\beta_{0}-\hat{\beta}\right)^{3}}{3 !\left(\beta^{*}\right)^{3}}$.

From Lemma 2, Lemma 3 and the fact that $\frac{1}{\left(\beta^{*}\right)^{3}}<\infty$, we can claim that $R_{n} \stackrel{p}{\rightarrow} 0$. Thus, by Lemma 1 to 4 , we have the result, that is, as $n \rightarrow \infty,-2 \log \mu(\underline{X}, \underline{Y})$ has the $\chi^{2}$ distribution with 1 degree of freedom. 


\subsection{Non-parametric test}

In this sub-section, we propose the non-parametric test, for testing $H_{0}: \beta=1$ against $H_{1}: \beta \neq 1$. The non-parametric estimate $\widetilde{\beta}$ of $\beta$ is obtained by replacing $\bar{F}(\cdot)$ by its empirical survival function, $\bar{F}_{n}$, in expression (4) and is given by

$$
\widetilde{\beta}=\left[-\frac{1}{n} \sum_{i=1}^{n} \log \left(1-\frac{\bar{F}_{n}\left(y_{i} ; \underline{\hat{\lambda}}\right)}{\bar{F}_{n}\left(x_{i} ; \underline{\hat{\lambda}}\right)}\right)\right]^{-1} .
$$

We note that, we do not have observations from $F(\cdot)$, baseline distribution, but we do have observation from the minimum (first failure), $X$. Suppose the c.d.f. of the minimum is $K(\cdot)=1-(1-F(\cdot))^{2}$, then by this assumption, we can write $F(\cdot)=1-\sqrt{(1-K(\cdot))}$. Let $K_{n}$ denote the empirical distribution function based on observations from $K(\cdot)$, then $F_{n}(\cdot)$ can be estimated as $1-\sqrt{\left(1-K_{n}(\cdot)\right)}$.

Theorem 2. Under independent and identical setup and as $n$ approaches infinity, the random variable $\sqrt{n}(\widetilde{\beta}-\beta)$ converge in distribution to a normal $N\left(0, \beta^{2}\right)$. That is

$$
\sqrt{n}(\widetilde{\beta}-\beta) \stackrel{D}{\rightarrow} Z \sim N\left(0, \beta^{2}\right) .
$$

Proof. For $i=1,2, \cdots, n$, consider

$$
\begin{gathered}
D_{i}=\left(\frac{\bar{F}_{n}\left(y_{i}\right)}{\bar{F}_{n}\left(x_{i}\right)}-\frac{\bar{F}\left(y_{i}\right)}{\bar{F}\left(x_{i}\right)}\right) \\
\leq\left|\frac{\bar{F}_{n}\left(y_{i}\right)}{\bar{F}_{n}\left(x_{i}\right)}-\frac{\bar{F}\left(y_{i}\right)}{\bar{F}\left(x_{i}\right)}\right| \\
\leq\left|\frac{\bar{F}_{n}\left(x_{i}\right)\left(\bar{F}_{n}\left(y_{i}\right)-\bar{F}\left(y_{i}\right)\right)-\bar{F}\left(y_{i}\right)\left(\bar{F}_{n}\left(x_{i}\right)-\bar{F}\left(x_{i}\right)\right)}{\left(\bar{F}_{n}\left(x_{i}\right) \bar{F}\left(x_{i}\right)\right.}\right| .
\end{gathered}
$$

Thus, by Glivenko-Cantelli theorem (Wasserman (2013)), we have, as $n \rightarrow \infty, \bar{F}_{n}(x) \stackrel{\text { a.s. }}{\rightarrow} \bar{F}(x)$ and hence, we can show that,

$$
\left(\frac{\bar{F}_{n}\left(y_{i}\right)}{\bar{F}_{n}\left(x_{i}\right)}-\frac{\bar{F}\left(y_{i}\right)}{\bar{F}\left(x_{i}\right)}\right) \stackrel{p}{\rightarrow} 0
$$

Hence,

$$
\left\{\frac{-1}{n} \sum_{i=1}^{n} \log \left(1-\frac{\bar{F}_{n}\left(y_{i}\right)}{\bar{F}_{n}\left(x_{i}\right)}\right)\right\}-\left\{\frac{-1}{n} \sum_{i=1}^{n} \log \left(1-\frac{\bar{F}\left(y_{i}\right)}{\bar{F}\left(x_{i}\right)}\right)\right\} \stackrel{p}{\rightarrow} 0,
$$

That is,

$$
\left(\frac{1}{\widetilde{\beta}}-\frac{1}{\widehat{\beta}}\right) \stackrel{p}{\rightarrow} 0
$$

As $0<\beta<\infty$, we have,

$$
(\widetilde{\beta}-\widehat{\beta}) \stackrel{p}{\rightarrow} 0,
$$

where, $\widehat{\beta}$ is as given in (4).

To obtain asymptotic distribution of $\widehat{\beta}$, we define, $Z_{i}=-\log \left(1-\frac{\bar{F}\left(y_{i}\right)}{\bar{F}\left(x_{i}\right)}\right), \quad \forall i=1,2, \ldots, n$. Since, $\left(X_{i}, Y_{i}\right)$ being i.i.d. pairs, we can claim that, $Z_{i}$ are also i.i.d. random variable. By CLT, we have,

$$
\bar{Z}=\frac{1}{n} \sum_{i=1}^{n} Z_{i} \stackrel{D}{\rightarrow} Z \sim N\left(E\left(Z_{1}\right), \frac{V\left(Z_{1}\right)}{n}\right) .
$$

By definition,

$$
E\left(Z_{1}\right)=\int_{x} \int_{y} z_{1} g(x, y) d y d x
$$




$$
=2 \beta \int_{\infty}^{0} \int_{\infty}^{x}\left\{-\log \left(1-\frac{\bar{F}\left(y_{i}\right)}{\bar{F}\left(x_{i}\right)}\right)\right\}\left(1-\frac{\bar{F}\left(y_{i}\right)}{\bar{F}\left(x_{i}\right)}\right)^{\beta-1} \frac{f(y)}{\bar{F}(x)} f(x) \bar{F}(x) d y d x .
$$

After putting $\left(1-\frac{\bar{F}\left(y_{i}\right)}{\bar{F}\left(x_{i}\right)}\right)=u$, we get,

$$
E\left(Z_{1}\right)=2 \beta \int_{\infty}^{0} \int_{1}^{0}\{-\log (u)\}(u)^{\beta-1} f(x) \bar{F}(x) d u d x=\beta \int_{1}^{0}\{-\log (u)\}(u)^{\beta-1} d u=\frac{1}{\beta} .
$$

Using similar argument, we can show that, $E\left(Z_{1}^{2}\right)=\frac{2}{\beta^{2}}$. Hence, the variance of $Z_{1}, V\left(Z_{1}\right)$ is $=\frac{1}{\beta^{2}}$. Thus, we claim that

$$
\bar{Z}\left(=\frac{1}{\hat{\beta}}\right) \stackrel{D}{\rightarrow} Z \sim N\left(\frac{1}{\beta}, \frac{1}{n \beta^{2}}\right)
$$

By Delta method, we have,

$$
\hat{\beta}=\left\{\frac{-1}{n} \sum_{i=1}^{n} \log \left(1-\frac{\bar{F}\left(y_{i}\right)}{\bar{F}\left(x_{i}\right)}\right)\right\} \stackrel{D}{\rightarrow} Z \sim N\left(\beta, \frac{\beta^{2}}{n}\right) .
$$

Thus, by law of convergence, we have the result.

\section{Simulation study}

In the present section, we carry out a simulation study to examine the power and the level attained by the LRT discussed in the Sub-section 3.1. In each case 10000 samples were generated for the different combinations of the sample sizes $(n)$ and the parameter values from the respective densities. There is evidence against $H_{0}$, if the value of the test statistic is $\geq 3.84$, which is the critical value at 0.05 of the $\chi^{2}$ distribution with 1 degree of freedom. In order to estimate power of LRT in case of Weibull, we generate 10000 samples of sample sizes, $n=10,30$ and 50 with parameters $(\lambda, \theta)$ as $(1,1),(1,2),(2,1),(0.5,1),(0.5,2)$ and $\beta$ as 0.5 , $0.8,1.5,1.8$ from the density given in $(7)$. To see the level attained by the test, we generated 10000 samples of sample sizes, $n=30,50,100$ with parameters $(\lambda, \theta)$ as $(1,1),(1,2),(2,1)$, $(0.5,1),(0.5,2)$ and $\beta=1$. We report the level attained in Table 1 and the estimated power of LRT for Weibull in Table 2. From Table 2, it is observed that the power improves as the sample size increases and as the parameter $\beta$ moves away from 1 . But we need a large sample size to attain 0.05 level. Similarly, in order to estimate power of LRT in case of LFR, we generate 10000 samples of sample sizes, $n=10,30$ and 50 with parameters $\left(\lambda_{1}, \lambda_{2}\right)$ as $(1,1)$, $(1,2),(2,1)$ and $\beta$ as $0.5,0.8,1.2,1.5,1.8$ from the density given in (10). To examine the level attained by the test, we generate 10000 samples of sample sizes, $=30,50,200,300,500$ with parameters $\left(\lambda_{1}, \lambda_{2}\right)$ as $(1,1),(1,2),(2,1)$ and $\beta=1$. We report the level attained in Table 3 and the estimated power of LRT for LFR in Table 4. From Table 4, it is observed that the power improves as the sample size increases and as the parameter $\beta$ moves away from 1 . To achieve 0.05 , the desired level of significance, we need a large sample size.

The simulation study of the non-parametric test, discussed in the Sub-section 3.2, is also presented. To see the estimated power and level attained, we generate 10000 samples for different combinations of sample sizes and parameter values from the respective densities. Under $H_{0}: \beta=1$, the distribution of test statistic $\sqrt{n}(\widetilde{\beta}-1)$ for testing $H_{0}: \beta=1$ against $H_{1}: \beta \neq 1$ is $N(0,1)$ and we use standard normal cutoff for the proposed non-parametric test procedure. Thus, we reject $H_{0}$, if the calculated value of test statistic $\geq 1.96$, the critical value at $5 \%$ of standard normal variate. We have observations from $K(\cdot)=1-(1-F(\cdot))^{m}$, the distribution function of first failure. Thus, we replace $F(\cdot)$ by $1-(1-K(\cdot))^{(1 / m)}$ and use the empirical distribution function based on $K(\cdot)$. To estimate power of the test with Weibull 
Table 1: Level attained by LRT in Weibull case for different sample sizes $(n)$.

\begin{tabular}{|c|c|c|c|c|c|}
\hline$\lambda$ & $\theta$ & $\beta$ & $n=30$ & $n=50$ & $n=100$ \\
\hline 1.0 & 1.0 & 1.0 & 0.057 & 0.053 & 0.047 \\
\hline 1.0 & 2.0 & 1.0 & 0.053 & 0.051 & 0.045 \\
\hline 2.0 & 1.0 & 1.0 & 0.056 & 0.052 & 0.048 \\
\hline 0.5 & 1.0 & 1.0 & 0.057 & 0.051 & 0.046 \\
\hline 0.5 & 2.0 & 1.0 & 0.055 & 0.049 & 0.047 \\
\hline
\end{tabular}

Table 2: Power of LRT in Weibull case for different sample sizes $(n)$.

\begin{tabular}{|c|c|c|c|c|c|}
\hline$\lambda$ & $\theta$ & $\beta$ & $n=10$ & $n=30$ & $n=50$ \\
\hline 1.0 & 1.0 & 0.5 & 0.869 & 0.960 & 0.997 \\
\hline 1.0 & 2.0 & 0.5 & 0.881 & 0.963 & 0.993 \\
\hline 2.0 & 1.0 & 0.5 & 0.871 & 0.959 & 0.999 \\
\hline 0.5 & 1.0 & 0.5 & 0.882 & 0.967 & 1.000 \\
\hline 0.5 & 2.0 & 0.5 & 0.878 & 0.965 & 0.999 \\
\hline 1.0 & 1.0 & 0.8 & 0.177 & 0.254 & 0.352 \\
\hline 1.0 & 2.0 & 0.8 & 0.199 & 0.258 & 0.383 \\
\hline 2.0 & 1.0 & 0.8 & 0.188 & 0.243 & 0.370 \\
\hline 0.5 & 1.0 & 0.8 & 0.183 & 0.238 & 0.393 \\
\hline 0.5 & 2.0 & 0.8 & 0.191 & 0.247 & 0.346 \\
\hline 1.0 & 1.0 & 1.5 & 0.415 & 0.571 & 0.808 \\
\hline 1.0 & 2.0 & 1.5 & 0.414 & 0.586 & 0.786 \\
\hline 2.0 & 1.0 & 1.5 & 0.405 & 0.597 & 0.822 \\
\hline 0.5 & 1.0 & 1.5 & 0.394 & 0.581 & 0.812 \\
\hline 0.5 & 2.0 & 1.5 & 0.393 & 0.557 & 0.814 \\
\hline 1.0 & 1.0 & 1.8 & 0.872 & 0.967 & 0.998 \\
\hline 1.0 & 2.0 & 1.8 & 0.871 & 0.975 & 1.000 \\
\hline 2.0 & 1.0 & 1.8 & 0.852 & 0.964 & 0.999 \\
\hline 0.5 & 1.0 & 1.8 & 0.876 & 0.968 & 0.997 \\
\hline 0.5 & 2.0 & 1.8 & 0.848 & 0.974 & 0.998 \\
\hline
\end{tabular}

distribution as baseline distribution, we generate 10000 samples of sample sizes $n=30,50$ and 100 with parameters $(\lambda, \theta)$ as $(1,1),(1,2),(2,1),(2,2),(0.5,1),(0.5,2)$ and $\beta$ as $0.5,0.8$, $1.5,1.8$ from the density given in (7). To examine the level attained, we generate 10000 samples of sample sizes $n=30,50,100$ and 200 with parameters $(\lambda, \theta)$ as $(1,1),(1,2),(2,1)$, $(0.5,1),(0.5,2)$ and $\beta=1$. In Tables 5 and 6 , respectively, report the level attained and the estimated power of the non-parametric test, with Weibull distribution as baseline distribution. From Table 6 it is observed that the power improves as the sample size increases and as the parameter $\beta$ moves away from 1. But we need a large sample size to attain 0.05 as level of significance. Similarly, in order to estimate power of the non-parametric test in case of LFR, we generate 10000 samples of sample sizes $n=10,30$ and 50 with parameters $\left(\lambda_{1}, \lambda_{2}\right)$ as $(1,1)$, $(1,2),(2,1),(2,2)$ and $\beta$ as $0.5,0.8,1.5,1.8$ from the density given in (10). To examine at the level attained by the test, we generate 10000 samples of sample sizes $n=30,50,200$ and 300 with parameters $\left(\lambda_{1}, \lambda_{2}\right)$ as $(1,1),(1,2),(2,1),(2,2)$ and $\beta=1$. We report the level attained and the estimated power of non-parametric test in Table 7 and Table 8 respectively, for LFR baseline distribution. Here also, it is observed that the power improves as the sample size increases and as the parameter $\beta$ moves away from 1 . We note that, to achieve the desired level of significance by the non-parametric test, we need a larger sample size as compare to the LRT. We have used R software for computation purpose. 
Table 3: Level attained by LRT in LFR case for different sample sizes $(n)$.

\begin{tabular}{|c|c|c|c|c|c|c|c|}
\hline$\lambda_{1}$ & $\lambda_{2}$ & $\beta$ & $n=30$ & $n=50$ & $n=200$ & $n=300$ & $n=500$ \\
\hline 1.0 & 1.0 & 1.0 & 0.140 & 0.150 & 0.100 & 0.058 & 0.048 \\
\hline 1.0 & 2.0 & 1.0 & 0.130 & 0.110 & 0.090 & 0.048 & 0.046 \\
\hline 2.0 & 1.0 & 1.0 & 0.160 & 0.113 & 0.087 & 0.053 & 0.047 \\
\hline
\end{tabular}

Table 4: Power of LRT in LFR case for different sample sizes $(n)$.

\begin{tabular}{|c|c|c|c|c|c|}
\hline$\lambda_{1}$ & $\lambda_{2}$ & $\beta$ & $n=10$ & $n=30$ & $n=50$ \\
\hline 1.0 & 1.0 & 0.5 & 0.562 & 0.950 & 1.000 \\
\hline 1.0 & 2.0 & 0.5 & 0.554 & 0.960 & 0.990 \\
\hline 2.0 & 1.0 & 0.5 & 0.523 & 0.910 & 0.990 \\
\hline 1.0 & 1.0 & 0.8 & 0.130 & 0.250 & 0.390 \\
\hline 1.0 & 2.0 & 0.8 & 0.160 & 0.280 & 0.450 \\
\hline 2.0 & 1.0 & 0.8 & 0.210 & 0.273 & 0.320 \\
\hline 1.0 & 1.0 & 1.5 & 0.430 & 0.660 & 0.830 \\
\hline 1.0 & 2.0 & 1.5 & 0.460 & 0.570 & 0.800 \\
\hline 2.0 & 1.0 & 1.5 & 0.370 & 0.660 & 0.810 \\
\hline 1.0 & 1.0 & 1.8 & 0.510 & 0.810 & 0.960 \\
\hline 1.0 & 2.0 & 1.8 & 0.600 & 0.840 & 0.916 \\
\hline 2.0 & 1.0 & 1.8 & 0.590 & 0.800 & 0.970 \\
\hline
\end{tabular}

Table 5: Level attained by non-parametric test in Weibull case for different sample sizes $(n)$.

\begin{tabular}{|c|c|c|c|c|c|c|}
\hline$\lambda$ & $\theta$ & $\beta$ & $n=30$ & $n=50$ & $n=100$ & $n=200$ \\
\hline 1.0 & 1.0 & 1.0 & 0.229 & 0.198 & 0.064 & 0.058 \\
\hline 1.0 & 2.0 & 1.0 & 0.234 & 0.199 & 0.068 & 0.057 \\
\hline 2.0 & 1.0 & 1.0 & 0.231 & 0.188 & 0.059 & 0.058 \\
\hline 2.0 & 2.0 & 1.0 & 0.261 & 0.191 & 0.061 & 0.056 \\
\hline 0.5 & 1.0 & 1.0 & 0.246 & 0.192 & 0.064 & 0.056 \\
\hline 0.5 & 2.0 & 1.0 & 0.262 & 0.194 & 0.068 & 0.058 \\
\hline
\end{tabular}


Table 6: Power of non-parametric test in Weibull case for different sample sizes $(n)$.

\begin{tabular}{|c|c|c|c|c|c|}
\hline$\lambda$ & $\theta$ & $\beta$ & $n=10$ & $n=30$ & $n=50$ \\
\hline 1.0 & 1.0 & 0.5 & 0.599 & 0.716 & 0.876 \\
\hline 1.0 & 2.0 & 0.5 & 0.612 & 0.723 & 0.885 \\
\hline 2.0 & 1.0 & 0.5 & 0.613 & 0.743 & 0.895 \\
\hline 2.0 & 2.0 & 0.5 & 0.628 & 0.761 & 0.894 \\
\hline 0.5 & 1.0 & 0.5 & 0.632 & 0.743 & 0.918 \\
\hline 0.5 & 2.0 & 0.5 & 0.627 & 0.732 & 0.874 \\
\hline 1.0 & 1.0 & 0.8 & 0.463 & 0.491 & 0.536 \\
\hline 1.0 & 2.0 & 0.8 & 0.451 & 0.489 & 0.535 \\
\hline 2.0 & 1.0 & 0.8 & 0.449 & 0.468 & 0.563 \\
\hline 2.0 & 2.0 & 0.8 & 0.487 & 0.476 & 0.529 \\
\hline 0.5 & 1.0 & 0.8 & 0.461 & 0.486 & 0.546 \\
\hline 0.5 & 2.0 & 0.8 & 0.453 & 0.489 & 0.556 \\
\hline 1.0 & 1.0 & 1.5 & 0.693 & 0.877 & 0.926 \\
\hline 1.0 & 2.0 & 1.5 & 0.685 & 0.868 & 0.925 \\
\hline 2.0 & 1.0 & 1.5 & 0.675 & 0.876 & 0.928 \\
\hline 2.0 & 2.0 & 1.5 & 0.689 & 0.874 & 0.928 \\
\hline 0.5 & 1.0 & 1.5 & 0.681 & 0.874 & 0.928 \\
\hline 0.5 & 2.0 & 1.5 & 0.702 & 0.873 & 0.927 \\
\hline 1.0 & 1.0 & 1.8 & 0.856 & 0.959 & 0.989 \\
\hline 1.0 & 2.0 & 1.8 & 0.837 & 0.955 & 0.988 \\
\hline 2.0 & 1.0 & 1.8 & 0.844 & 0.957 & 0.988 \\
\hline 2.0 & 2.0 & 1.8 & 0.875 & 0.955 & 0.989 \\
\hline 0.5 & 1.0 & 1.8 & 0.896 & 0.955 & 0.989 \\
\hline 0.5 & 2.0 & 1.8 & 0.884 & 0.955 & 0.988 \\
\hline
\end{tabular}

Table 7: Level attained by non-parametric test in LFR case for different sample sizes $(n)$.

\begin{tabular}{|c|c|c|c|c|c|c|}
\hline$\lambda_{1}$ & $\lambda_{2}$ & $\beta$ & $n=30$ & $n=50$ & $n=200$ & $n=300$ \\
\hline 1.0 & 1.0 & 1.0 & 0.203 & 0.117 & 0.069 & 0.054 \\
\hline 1.0 & 2.0 & 1.0 & 0.240 & 0.130 & 0.068 & 0.053 \\
\hline 2.0 & 1.0 & 1.0 & 0.239 & 0.131 & 0.063 & 0.054 \\
\hline 2.0 & 2.0 & 1.0 & 0.240 & 0.130 & 0.065 & 0.054 \\
\hline
\end{tabular}

\section{Illustrations}

In present section, we report two illustrations with two data sets namely appendectomy in Australian twins and motors data, to see how the proposed test procedures work.

\subsection{Appendectomy in Australian twins}

The Australian Twins Data (Duffy, Martin, and Mathews (1990)) was derived from a questionnaire mailed to 5967 twin pairs, over the age of 18 years registered with the Australian Twin Registry during the period from November 1980 to March 1982. Subjects were asked if they had undergone appendectomy and at what age the procedure was performed (onset). A total of 3,808 complete pairs returned the questionnaire. We have taken only the pairs of twins who had undergone appendectomy. There were 339 such pairs. Out of the 339 pairs of twins we discarded 6 pairs of twins for whom the appendectomy was performed at the same age, treating these observation as ties. 
Table 8: Power of non-parametric test in LFR case for different sample sizes $(n)$.

\begin{tabular}{|c|c|c|c|c|c|}
\hline$\lambda_{1}$ & $\lambda_{2}$ & $\beta$ & $n=10$ & $n=30$ & $n=50$ \\
\hline 1.0 & 1.0 & 0.5 & 0.632 & 0.770 & 0.855 \\
\hline 1.0 & 2.0 & 0.5 & 0.619 & 0.719 & 0.865 \\
\hline 2.0 & 1.0 & 0.5 & 0.653 & 0.768 & 0.866 \\
\hline 2.0 & 2.0 & 0.5 & 0.628 & 0.791 & 0.834 \\
\hline 1.0 & 1.0 & 0.8 & 0.474 & 0.503 & 0.579 \\
\hline 1.0 & 2.0 & 0.8 & 0.428 & 0.567 & 0.593 \\
\hline 2.0 & 1.0 & 0.8 & 0.476 & 0.561 & 0.596 \\
\hline 2.0 & 2.0 & 0.8 & 0.473 & 0.566 & 0.599 \\
\hline 1.0 & 1.0 & 1.5 & 0.851 & 0.922 & 0.605 \\
\hline 1.0 & 2.0 & 1.5 & 0.885 & 0.925 & 0.985 \\
\hline 2.0 & 1.0 & 1.5 & 0.853 & 0.926 & 0.983 \\
\hline 2.0 & 2.0 & 1.5 & 0.884 & 0.926 & 0.985 \\
\hline 1.0 & 1.0 & 1.8 & 0.954 & 0.992 & 1.000 \\
\hline 1.0 & 2.0 & 1.8 & 0.966 & 0.991 & 0.999 \\
\hline 2.0 & 1.0 & 1.8 & 0.952 & 0.987 & 1.000 \\
\hline 2.0 & 2.0 & 1.8 & 0.961 & 0.984 & 1.000 \\
\hline
\end{tabular}

We consider a twin pair as a two component parallel system with the age at appendectomy as the time to failure. Generally it is assumed that the twins are associated with each other as far as their health problems are concerned. Our interest is to test whether the age of appendectomy of one individual is associated with the age of the other. We first apply the Wilcoxon-Mann-Whitney type test proposed by Deshpande et al. (2010) to test for the load sharing effect. Here, $H_{0}$ is that times of appendectomy of twins are independent and $H_{1}$ is that the time of appendectomy of one individual is associated with that of the other. The value of the test statistic is -5.8854 and the $\mathrm{p}$-value $=0.0001$, which shows evidence in favor of $H_{1}$. Thus we conclude that the time of appendectomy of one twin has an effect on the time of appendectomy of the other.

Kolmogorov-Smirnov (KS) type test showed that the LFR distribution is appropriate distribution for the component failure time. This test is conservative as we estimated the unknown parameters by the proposed two step procedure. In case of Weibull the two stage estimates of $\lambda, \theta$ and $\beta$ are 2.0849, 0.0012 and 0.8005 respectively and in case of LFR the two stage estimates of $\lambda_{1}, \lambda_{2}$ and $\beta$ are $0.0028,0.0030$ and 0.7875 respectively.

In order to investigate whether the load sharing model proposed by Sutar and Naik-Nimbalkar (2016) provides a better fit to the data than the model proposed by Deshpande et al. (2010), we use Akaike Information Criteria (AIC) (Akaike (1974)) and Bayesian Information Criteria (BIC) (Ghosh, Delampady, and Samanta (2007)). We refer to the model proposed by Deshpande et al. (2010) as proportional conditional hazard rate (PCHR) model. The AIC and $\mathrm{BIC}$ are defined as

$$
A I C=-2 \log L(\cdot)+2 p, \quad B I C=-2 \log L(\cdot)+p \log (n)
$$

where $p$ represents number of parameters of the model, $n$ represents number of data points and $L(\cdot)$ is the maximized value of the likelihood function for the estimated model.

From Table 9, it is clear that LFR model is preferable for the Australian Twins Data. Table 10 shows the AIC and BIC values for the proposed PCRHR and PCHR models with initial component lifetimes as Weibull and as LFR.

From Table 10, we see that, PCRHR model is more appropriate than the PCHR model for the Australian Twins Data. It also shows that the LFR distribution with the PCRHR model gives a better fit. 
Table 9: AIC and BIC values for Australian Twins Data for Weibull and LFR.

\begin{tabular}{|c|c|c|}
\hline Distribution & AIC values & BIC values \\
\hline Weibull & 5272.95 & 5281.95 \\
\hline LFR & 5226.10 & 5235.11 \\
\hline
\end{tabular}

Table 10: AIC and BIC values for Australian Twins Data to compare PCHR and PCRHR Model.

\begin{tabular}{|c|c|c|c|}
\hline Model & Distribution & AIC values & BIC values \\
\hline PCHR & Weibull & 17682.21 & 17693.64 \\
\hline PCRHR & Weibull & 7691.19 & 7702.61 \\
\hline PCHR & LFR & 5824.63 & 5836.05 \\
\hline PCRHR & LFR & 743.26 & 754.69 \\
\hline
\end{tabular}

We also apply the LRT described in Section 3.1 to test the load sharing effect. The value of LRT statistic is 20.6042, which is significant both at $1 \%$ and $5 \%$ level. Hence we conclude that for a pair, the age of appendectomy of one twin is associated with that of the other twin.

\subsection{Motors data}

These data are taken from Reliability Edge Home (Home (2003)). The data set represents a life test on 18 parallel systems consisting of two electric motors operating continuously. The data consist of the failure times of both the motors along with their identification labels (A and B) for each system. We consider the ordered component failure times for each system. Here, we wish to test whether failures of motors occur independently. Sutar and Naik-Nimbalkar (2016) have shown that the failure of a motor affects the working motor or there exists a load sharing phenomenon. They have also shown that the PCRHR model, proposed by Sutar and Naik-Nimbalkar (2016), is more appropriate than the PCHR model, proposed by Deshpande et al. (2010), for the motors data in case of both Weibull and LFR distributions as the baseline distributions of the components.

We also apply LRT to the same data set. The value of LRT statistic is 6.4038 , which is significant both at $1 \%$ and $5 \%$ level. Hence we may conclude that the failure of a motor affects the working motor. But the value of non-parametric test statistic is 1.7819 which is not significant both at $1 \%$ and $5 \%$ level, since it is also seen from the simulation study that it reqires larger sample size.

\section{Conclusions}

We considered the load sharing model proposed by Sutar and Naik-Nimbalkar (2016). We have proposed a likelihood ratio test and a non-parametric test for tesing the load sharing effect observed in a two component parallel load sharing system. We report the simulation study to check the performance of the proposed test procedures for a two component parallel load sharing system with baseline distribution as Weibull distribution as well as linear failure rate distribution. The simulation study shows that the proposed test procedure is quite satisfactory. To see the practical applicability of the proposed tests, we report the two illustrations.

\section{Acknowledgment}

Author is grateful to the Shivaji University, Kolhapur, India for the financial support in the form of 'Research Initiation Scheme' (Ref:O.W./S.U./C.A.U.D.Section/1067/15th March, 
2018).

\section{References}

Akaike H (1974). "A New Look at the Statistical Model Identification.” pp. 215-222.

Amari SV, Bergman R (2008). "Reliability Analysis of k-out-of-n Load-sharing Systems." In 2008 Annual Reliability and Maintainability Symposium, pp. 440-445. IEEE.

Amari SV, Misra KB, Pham H (2006). "Reliability Analysis of Tampered Failure Rate Loadsharing k-out-of-n: G Systems." In Proc. 12th ISSAT Int. Conf. on Reliability and Quality in Design, Honolulu, Hawaii, pp. 30-35. Citeseer.

Birnbaum ZW, Saunders SC (1958). "A Statistical Model for Life-length of Materials." Journal of the American Statistical Association, 53(281), 151-160.

Coleman BD (1958). "Statistics and Time Dependence of Mechanical Breakdown in Fibers." Journal of Applied Physics, 29(6), 968-983.

Cramer E, Kamps U (1996). "Sequential Order Statistics and k-out-of-n Systems with Sequentially Adjusted Failure Rates." Annals of the Institute of Statistical Mathematics, 48(3), $535-549$.

Cramer E, Kamps U (2001). "Sequential k-out-of-n Systems, Advances in Reliability (Handbook Statist. 20)."

Cramer E, Kamps U (2003). "Marginal Distributions of Sequential and Generalized Order Statistics." Metrika, 58(3), 293-310.

Cramer H (1946). "Mathematical Methods of Statistics." Princeton U. Press, Princeton, p. 500 .

Daniels HE (1945). "The Statistical Theory of the Strength of Bundles of Threads. I." Proceedings of the Royal Society of London. Series A. Mathematical and Physical Sciences, 183(995), 405-435.

Deshpande JV, Dewan I, Naik-Nimbalkar UV (2010). "A Family of Distributions to Model Load Sharing Systems." Journal of Statistical Planning and Inference, 140(6), 1441-1451.

Dewan I, Naik-Nimbalkar UV (2010). Load-sharing Systems. Wiley Online Library.

Drummond H, Vázquez E, Sánchez-Colón S, Martinez-Gómez M, Hudson R (2000). "Competition for Milk in the Domestic Rabbit: Survivors Benefit from Littermate Deaths." Ethology, 106(6), 511-526.

Duffy DL, Martin NG, Mathews JD (1990). "Appendectomy in Australian Twins." American Journal of Human Genetics, 47(3), 590.

Durham SD, Lynch JD (2000). "A Threshold Representation for the Strength Distribution of a Complex Load Sharing System." Journal of Statistical Planning and Inference, 83, 25-46.

Ghosh JK, Delampady M, Samanta T (2007). An Introduction to Bayesian Analysis: Theory and Methods. Springer Science \& Business Media.

Gross AJ, Clark VA, Liu V (1971). "Estimation of Survival Parameters when One of Two Organs Must Function for Survival." Biometrics, pp. 369-377.

Gurov SV, Utkin LV (2014). "A Load-share Reliability Model under the Changeable Piecewise Smooth Load." Journal of Quality and Reliability Engineering, 2014. 
Hollander M, Peña EA (1995). "Dynamic Reliability Models with Conditional Proportional Hazards." Lifetime Data Analysis, 1(4), 377-401.

Home RE (2003). "Reliability Edge Home." Quarter 2, 3. URL www.reliasoft.com.

Jain M, Gupta R (2012). "Load Sharing M-out of-N: G System with Non-identical Components Subject to Common Cause Failure." International Journal of Mathematics in Operational Research, 4(5), 586-605.

Kale BK (2005). A First Course on Parametric Inference. Alpha Science Int'l Ltd.

Kamps U (1995). "A Concept of Generalized Order Statistics." Journal of Statistical Planning and Inference, 48(1), 1-23.

Kim H, Kvam PH (2004). "Reliability Estimation Based on System Data with an Unknown Load Share Rule." Lifetime Data Analysis, 10(1), 83-94.

Kim KO (2018). "Optimal Number of Components in a Load-sharing System for Maximizing Reliability." Journal of the Korean Statistical Society, 47(1), 32-40.

Krivtsov V, Amari S, Gurevich V (2018). "Load Sharing in Series Configuration." Quality and Reliability Engineering International, 34(1), 15-26.

Kvam PH, Pena EA (2005). "Estimating Load-sharing Properties in a Dynamic Reliability System." Journal of the American Statistical Association, 100(469), 262-272.

Liu H (1998). "Reliability of a Load-sharing k-out-of-n: G System: Non-iid Components with Arbitrary Distributions." IEEE Transactions on Reliability, 47(3), 279-284.

Lynch JD (1999). "On the Joint Distribution of Component Failures for Monotone Loadsharing Systems." Journal of Statistical Planning and Inference, 78(1-2), 13-21.

McCool JI (2006). "Testing for Dependency of Failure Times in Life Testing." Technometrics, 48(1), 41-48.

Mohammad R, Kalam A, Amari SV (2013). "Reliability of Load-sharing Systems Subject to Proportional Hazards Model." pp. 1-5.

Peña EA (2006). "Dynamic Modelling and Statistical Analysis of Event Times." Statistical science: a review journal of the Institute of Mathematical Statistics, 21(4), 1.

Phoenix SL (1978). "The Asymptotic Time to Failure of a Mechanical System of Parallel Members." SIAM Journal on Applied Mathematics, 34(2), 227-246.

Rosen BW (1964). "Tensile Failure of Fibrous Composites." AIAA journal, 2(11), 1985-1991.

Singh B, Gupta PK (2012). "Load-sharing System Model and Its Application to the Real Data Set." Mathematics and Computers in Simulation, 82(9), 1615-1629.

Singpurwalla ND (1995). "Survival in Dynamic Environments." Statistical science, pp. 86-103.

Suprasad AV, Krishna MB, Hoang P (2008). "Tampered Failure Rate Load-sharing Systems: Status and Perspectives." In Handbook of Performability Engineering, pp. 291-308. Springer.

Sutar S, Naik-Nimbalkar UV (2019). "A Load Share Model for Non-identical Components of a k-out-of-m System." Applied Mathematical Modelling, 72, 486-498.

Sutar SS, Naik-Nimbalkar UV (2014). "Accelerated Failure Time Models for Load Sharing Systems." IEEE Transactions on Reliability, 63(3), 706-714. 
Sutar SS, Naik-Nimbalkar UV (2016). "A Model for k-out-of-m Load-sharing Systems." Communications in Statistics-Theory and Methods, 45(20), 5946-5960.

Wasserman L (2013). All of Statistics: a Concise Course in Statistical Inference. Springer Science \& Business Media.

Yang C, Zeng S, Guo J (2015). "Reliability Analysis of Load-sharing-out-of-system Considering Component Degradation." Mathematical Problems in Engineering, 2015.

\author{
Affiliation: \\ Santosh Shashikant Sutar \\ Yashwantrao Chavan School of Rural Development, \\ Shivaji University, Kolhapur, \\ Maharashtra, India - 416004. \\ E-mail: sutarssantosh@gmail.com \\ URL: https://http://www.unishivaji.ac.in/
}

\section{Austrian Journal of Statistics}

published by the Austrian Society of Statistics

Volume 50

January 2021 http://www.ajs.or.at/

http://www.osg.or.at/

Submitted: 2019-05-28

Accepted: 2019-09-18 